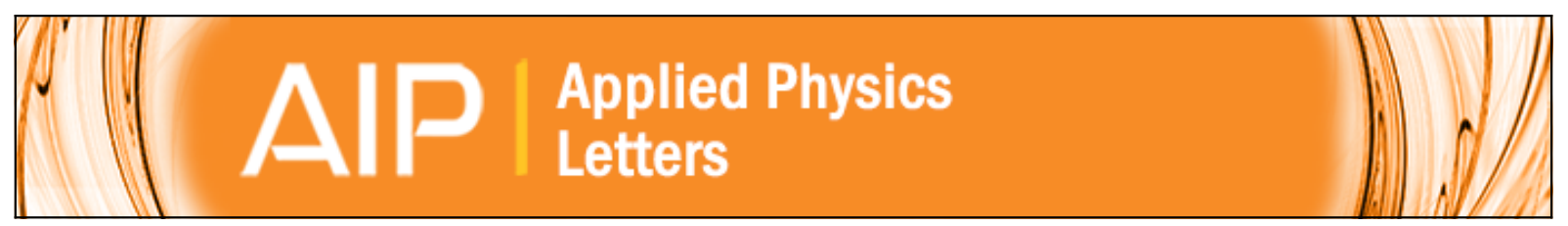

\title{
Experimental demonstration of superdirective dielectric antenna
}

Alexander E. Krasnok, Dmitry S. Filonov, Constantin R. Simovski, Yuri S. Kivshar, and Pavel A. Belov

Citation: Applied Physics Letters 104, 133502 (2014); doi: 10.1063/1.4869817

View online: http://dx.doi.org/10.1063/1.4869817

View Table of Contents: http://scitation.aip.org/content/aip/journal/apl/104/13?ver=pdfcov

Published by the AIP Publishing

\section{Articles you may be interested in}

Terahertz antenna based on graphene

J. Appl. Phys. 107, 104313 (2010); 10.1063/1.3427536

An antenna-coupled bolometer with an integrated microstrip bandpass filter

Appl. Phys. Lett. 86, 114103 (2005); 10.1063/1.1879115

High-directivity planar antenna using controllable photonic bandgap material at microwave frequencies Appl. Phys. Lett. 78, 4196 (2001); 10.1063/1.1382853

Photonic crystal-based resonant antenna with a very high directivity

J. Appl. Phys. 87, 603 (2000); 10.1063/1.371905

Light scattering from dipole and quadrupole nanoshell antennas

Appl. Phys. Lett. 75, 1063 (1999); 10.1063/1.124597

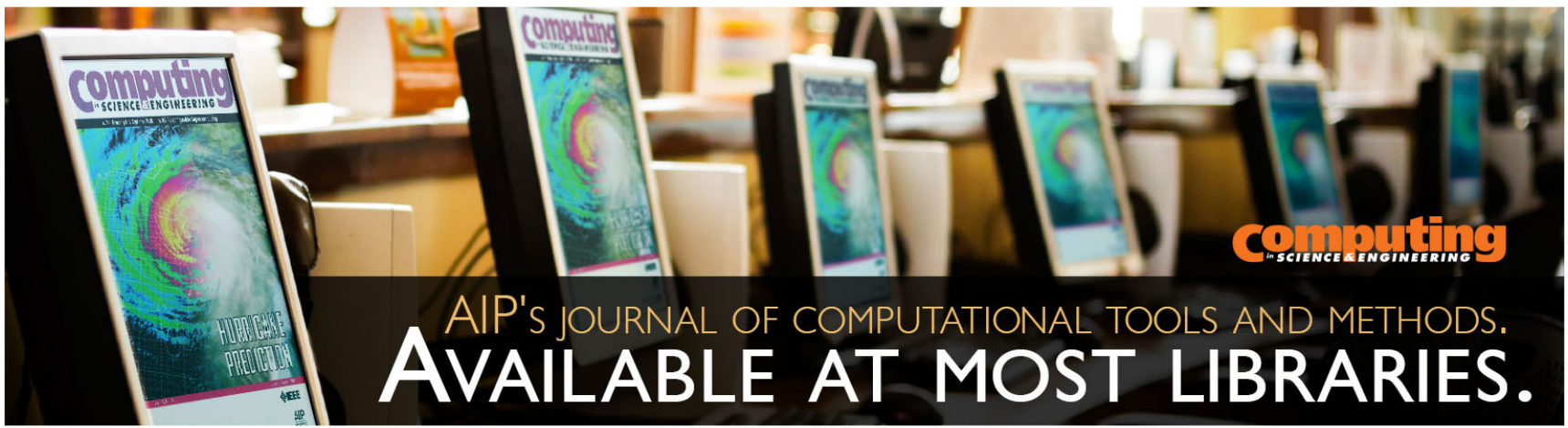




\title{
Experimental demonstration of superdirective dielectric antenna
}

\author{
Alexander E. Krasnok, ${ }^{1}$ Dmitry S. Filonov, ${ }^{1}$ Constantin R. Simovski, ${ }^{1,2}$ Yuri S. Kivshar, ${ }^{1,3}$ \\ and Pavel A. Belov ${ }^{1}$ \\ ${ }^{1}$ National Research University of Information Technologies, Mechanics and Optics (ITMO), \\ St. Petersburg 197101, Russia \\ ${ }^{2}$ Aalto University, School of Electric and Electronic Engineering, Aalto FI76000, Finland \\ ${ }^{3}$ Nonlinear Physics Center, Research School of Physics and Engineering, Australian National University, \\ Canberra ACT 0200, Australia
}

(Received 6 February 2014; accepted 16 March 2014; published online 31 March 2014)

\begin{abstract}
We propose and demonstrate experimentally a simple approach for achieving superdirectivity of emitted radiation for electrically small antennas based on a spherical dielectric resonator with a notch excited by a dipole source. Superdirectivity is achieved without using complex antenna arrays and for a wide range of frequencies. We also demonstrate the steering effect for a subwavelength displacement of the source. Finally, unlike previously known superdirective antennas, our design has significantly smaller losses, at the operation frequency radiation efficiency attains $80 \%$, and matching holds in the 3\%-wide frequency band without any special matching technique. (C) 2014 AIP Publishing LLC. [http://dx.doi.org/10.1063/1.4869817]
\end{abstract}

Despite a long history of classical antennas, ${ }^{1}$ dielectric antennas have been studied insufficiently, the now their investigation is stimulated by a rapid progress in optical communication systems which require a new type of compact nanoantennas implemented on a chip., ${ }^{2,3}$ Electrically small antennas, ${ }^{4-6}$ dielectric or metallic, possess weak directionality of radiation which is usually close to that of a point dipole. ${ }^{1}$ At microwaves, the high directionality is usually achieved utilizing the aperture antennas (e.g., a lens or a horn), the phased antenna arrays, the Yagi-Uda antennas, and leaky wave antennas. ${ }^{1}$ However, such antennas have the size larger than the radiation wavelength $\lambda_{0}$, and individual elements of these arrays are not electrically small.

High directionality of electrically small antennas can be also achieved; however, in this case the theory requires rapidly oscillating currents in the subwavelength area, which lead to the excitation of higher multipole modes in the antenna which may result in the high directionality in spite of the electrically small volume of the antenna, which is then called superdirective.

Superdirectivity is an important physical property which can be defined as significant enhancement of directionality of an electrically small antenna system as compared to the directionality of a point dipole. ${ }^{1,7-10}$ All previously known designs of superdirective antennas were based on discrete arrays of radiating dipole antennas with rather weird distribution of radiating currents over the array ${ }^{11-13}$ or were based on arrays of small resonant scatterers realizing so-called metamaterials. ${ }^{14-17}$ Intrinsic drawbacks of the mentioned designs are ultra-narrow frequency range, high dissipation, extreme sensitivity to perturbations, unstable operation, and challenging fabrication tolerances. Superdirective antennas are applied in space communications and radioastronomy, though they could in principle find many other applications if one gets rid of their drawbacks.

In this Letter, we propose and demonstrate experimentally a superdirective dielectric antenna, realizing at microwaves the principles recently suggested theoretically for optical nanoantennas in Ref. 18. A general realization of a superdirective antenna is based on a spherical dielectric resonator with a notch as depicted in Fig. 1. The feeding of antenna is performed by a coaxial cable with a short wire dipole located inside the notch. The size $R_{s}$ and refractive index $n$ of the spherical dielectric resonator are determined by the existence of lower resonator mode, corresponding to the magnetic Mie resonance

$$
n R_{s} / \lambda_{0} \approx 1,
$$

and it is clear that the resonator should be made of a highpermittivity material like ceramics. In the case of homogeneous excitation, lower-order multipole modes of such the resonator are excited while the contribution of higher-order modes is weak. ${ }^{19-21}$ However, making a small notch at the surface of the resonator and introducing a dipole source inside it breaks the spherical symmetry and allows the resonant excitation of higher-order multipoles. Higher-order multipole modes have directive radiation patterns and, therefore, their excitation provides high directionality of this resonator antenna. As a material of our resonator, we employ $\mathrm{MgO}-\mathrm{TiO}_{2}$ ceramic spheres ${ }^{21}$ characterized at microwaves by dielectric permittivity close to 16 and low loss tangent 1.12-1.17 $10^{-4}$. Using the condition (1), the spherical resonator made of $\mathrm{MgO}-\mathrm{TiO}_{2}$ with the radius $R_{\mathrm{S}}=5 \mathrm{~mm}$ should have lowest Mie resonance at the frequency close to 14 GHz. In accordance with Ref. 18, the superdirectivity is expected at slightly $(10 \%-15 \%)$ higher frequencies, i.e., near $16 \mathrm{GHz}$. The radius of the semi-spherical notch is equal to $R_{\mathrm{n}}=2 \mathrm{~mm}$, the length of the dipole source is $L=1.5 \mathrm{~mm}$, arms of length $L / 2$ are connected to the core and shell of a feeding coaxial cable.

We have performed numerical simulations of this antenna system in CST Microwave Studio. To characterize the antenna properties, we calculated the directivity $D$ and radiation efficiency $\eta$ defined as 


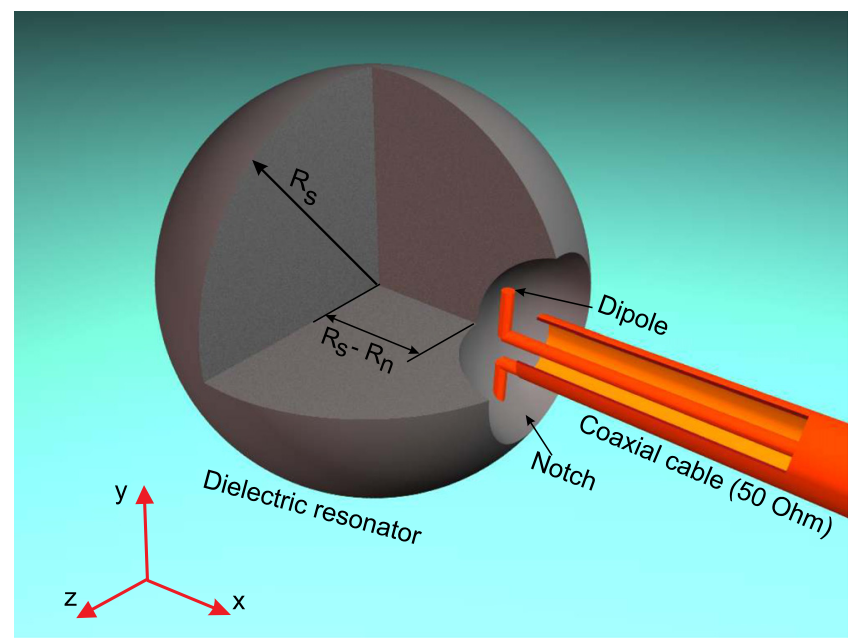

FIG. 1. A conceptional view of the superdirective antenna based on a dielectric notched resonator. A short wire dipole fed by a coaxial cable is employed as a source. The dipole is located inside the notch.

$$
D=\frac{4 \pi \operatorname{Max}[p(\theta, \varphi)]}{P_{\mathrm{rad}}}, \quad \eta=\frac{P_{\mathrm{rad}}}{P_{\mathrm{rad}}+P_{\mathrm{loss}}},
$$

where $P_{\text {rad }}=\int_{\Omega} p(\theta, \varphi) d \Omega$ is a total power radiated in the far field, $P_{\text {loss }}$ is a the power of dissipative losses in antenna material, $\operatorname{Max}[p(\theta, \varphi)]$ is the power flux density radiated in the direction of maximal flux density, $\theta$ and $\varphi$ are the angles of conventional spherical system, $d \Omega$ is the elementary solid angle. Figure 2 shows the dependencies of $D$ and $\eta$ on the wavelength for the chosen geometric parameters of the antenna. The directivity has the maximum $\left(D_{\max }=11\right)$ at frequency $16.4 \mathrm{GHz}$ where the total size of the antenna is close to $0.38 \lambda$. The mode analysis (see in Ref. 18), shows that in this case the local field distribution inside a resonator corresponds to noticeable higher-order multipoles. The observed directivity is close to that of an all-dielectric Yagi-Uda antenna with maximal size $2 \lambda$ which is five times larger. ${ }^{21}$ The value of radiation efficiency at frequency 16.7 $\mathrm{GHz}$ where the directivity equals to 10 is equal 0.8 . In comparison to conventional superdirective antennas, our design has significantly smaller dissipative losses that achieved by using the low losses dielectric material and avoiding resistive loads. ${ }^{11-13}$ Next, we have measured the radiation patterns of

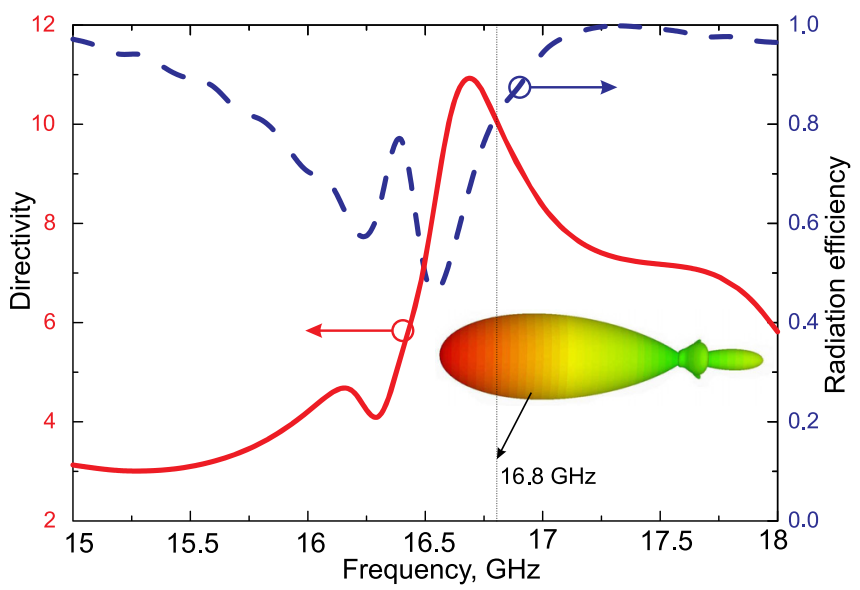

FIG. 2. Directivity and radiation efficiency of the superdirective antenna obtained from the CST numerical simulations.

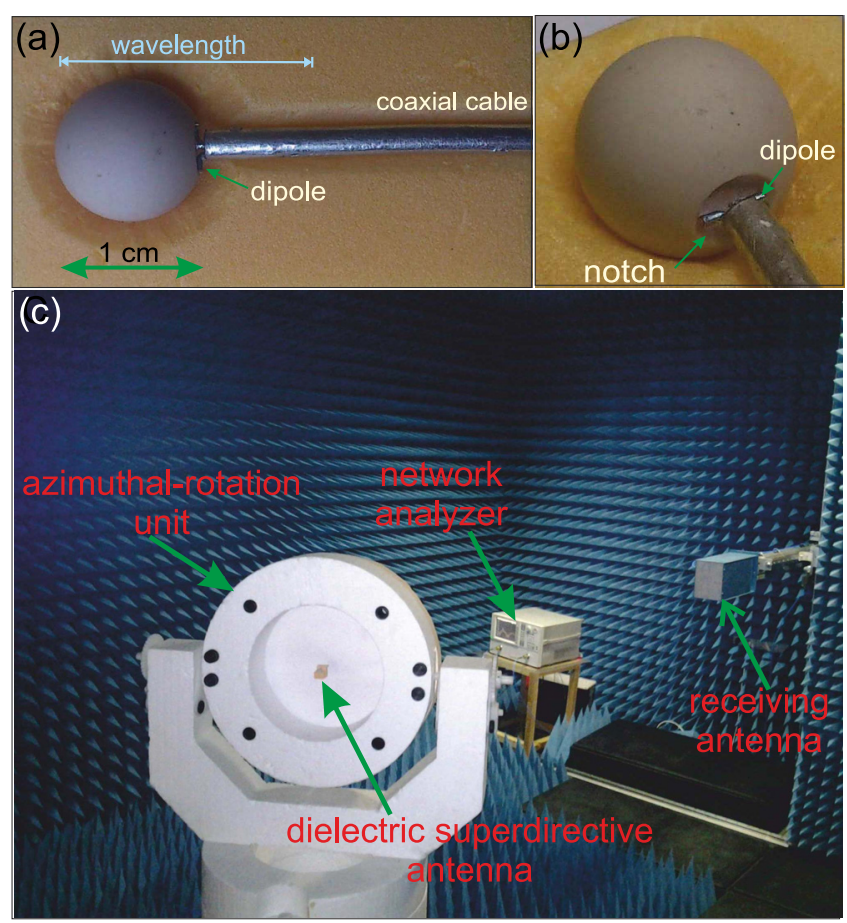

FIG. 3. Photographs of (a) top view and (b) perspective view of a superdirective antenna. (c) Image of the experimental setup for measuring of power patterns.

the antenna in the anechoic chamber. The photographs of top view and perspective view of this superdirective dielectric antenna are presented in Figs. 3(a) and 3(b). Figure 3(c) shows the general view of the experimental setup. Radiating power was measured by a standard ultra-wideband towermount antenna (TMA) probe capable to move in the far field zone of the tested antenna. Styrofoam material with the dielectric permittivity close to 1 is used to fix the TMA probe in the azimuthal-rotation unit. The results of experimental investigations and numerical simulations of the radiation pattern in both $E$ - and $H$-planes for the symmetrical dipole arrangement in the notch are summarized in Figs. 4(a) and 4(b). Radiation patterns in both planes are narrow beams with a lobe angle about $35^{\circ}$. Experimental values for the directivity in the $E$ - and $H$-planes are equal to 5.9 and 8.4, respectively. The numerical values of these quantities are equal to 6.8 and 8.1. So the experimental data agree well with the numerical results. The small difference can be explained by the slight asymmetry of the wire dipole.

We have found another exciting property of the proposed antenna: a small displacement of the wire dipole in the horizontal plane $(x-z)$ (see in Fig. 1) leads to a noticeable turn of the main radiation beam without damage for the directionality. Since the source still keeps inside the electrically small notch, this effect means the subwavelength sensitivity of the radiation pattern to the exact position of the source phase center. Our numerical calculations show that this antenna geometry allows to radiation pattern rotation up to $25^{\circ}$ with insignificant increase of dissipative losses. Experimental and numerical demonstration of this beam steering effect are presented in Figs. 4(c) and 4(d). For the chosen geometry of antenna, displacement of the dipole by $0.5 \mathrm{~mm}(\lambda / 37)$ leads to the turn of the pattern about $10^{\circ}$. This effect along with the 


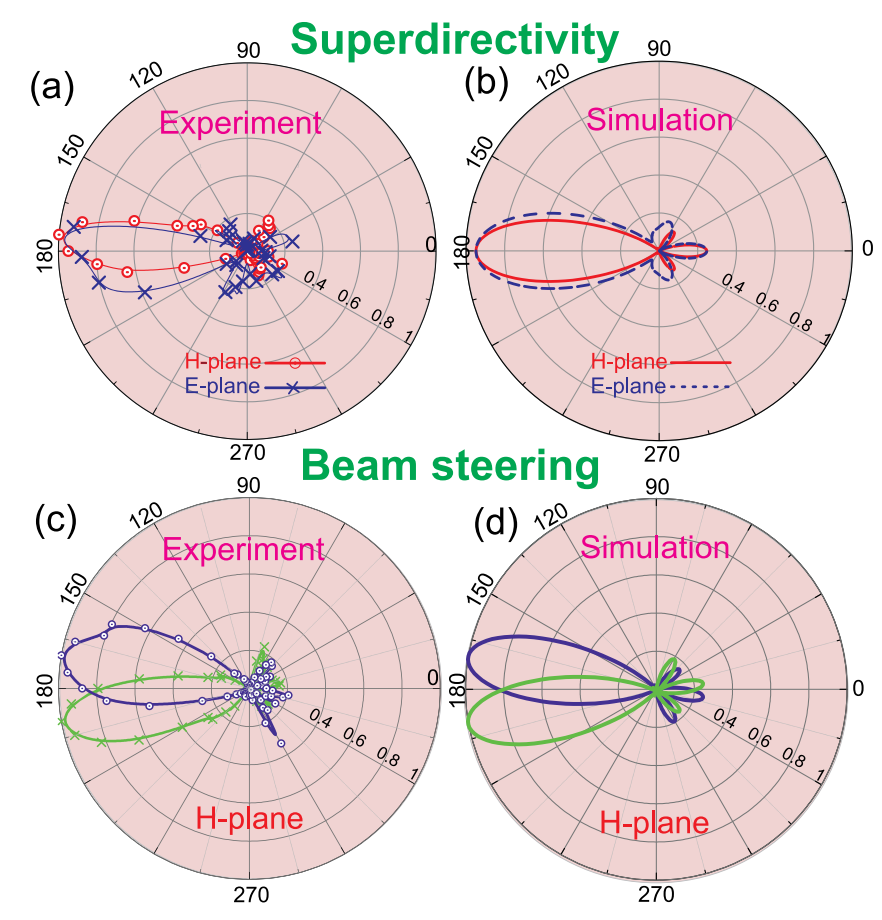

FIG. 4. Experimental (a) and numerical (b) radiation patterns of the antenna in both $E$ - and $H$-planes for the symmetrical dipole arrangement at the frequency $16.8 \mathrm{GHz}$. The crosses and circles correspond to the experimental data. Experimental (c) and numerical (d) demonstration of beam steering effect, displacement of dipole is equal $0.5 \mathrm{~mm}$.

high radiation efficiency that equals $\eta=0.8-0.9$ in the $3 \%$ wide operation band $(16.6-17.1 \mathrm{GHz})$ makes our superdirective antenna promising for many practical applications. Note that the similar beam steering is observed in the lens antenna, including based on gradient refractive index metamaterials. ${ }^{22}$ However, our design is not the lens antenna because of its subwavelength dimensions.

It is important to discuss the matching of our antenna with the coaxial cable which is a prerequisite of this high efficiency. Though the dipole length in the operation band is as small as $L \approx \lambda / 12$, it has rather good matching. Its asymmetry is so small that practically, no currents are induced on the cable shell. This self-matching effect can be described as tuning the short wire dipole and increasing its radiation resistance by the dielectric resonator. The tuning effect results from an inductive impedance induced in the dipole by the polarization of the dielectric resonator. Also, the polarized resonator induces in the dipole antenna an additional radiation resistance. The input resistance of the dipole increases and becomes close to the wave impedance of the cable. The input reactance due to the tuning effect becomes much smaller than this resistance. Thus, we obtain a good matching.

Let us derive a ratio between the power radiated by a short dipole in presence of the notched resonator and in its absence using the induced impedance method. ${ }^{1}$ In the approximate version of this method, referring to electrically small dipole antennas, the power radiated by a short dipole in an arbitrary inhomogeneous environment is proportional to the imaginary part of the Green's function of this environment referred to the dipole center $\mathbf{r}_{0}$. Namely, $P_{\text {rad }} \sim \operatorname{Im}\left[G_{y y}\left(\mathbf{r}_{0}, \mathbf{r}_{0}, \omega\right)\right]$, where $G_{y y}$ is the co-component of dyadic Green's function (see our coordinate system in Fig. 1). Let us link this Green's function with the dipole input impedance. It is known that the current distribution along a short wire dipole is nearly triangular. ${ }^{23,24}$ If $I_{0}$ is the amplitude of the current at the dipole center, the expression for the radiation impedance $Z_{\mathrm{rad}}$ can be written as $Z_{\mathrm{rad}}=E_{y}\left(\mathbf{r}_{0}\right) L / 2 I_{0}$, where $E_{y}\left(\mathbf{r}_{0}\right)$ is $y$-component of the electric field produced by the radiating dipole and its polarized environment at $\mathbf{r}_{0}$. Coefficient $1 / 2$ results from the triangular current distribution which decreases twice the effective length $L_{\text {eff }}$ of the dipole compared to $L$.

On the other hand, from definition of Green's function we have $E_{y}\left(\mathbf{r}_{\mathbf{0}}\right)=\omega^{2} \mu_{0} G_{y y}\left(\mathbf{r}_{0}, \mathbf{r}_{0}, \omega\right) d$, where $d$ is the radiating dipole moment $d=-j I_{0} L_{\text {eff }} / \omega=-j I_{0} L / 2 \omega$. Of course, $G_{y y}\left(\mathbf{r}_{0}, \mathbf{r}, \omega\right)$ has singularity at $\mathbf{r}=\mathbf{r}_{0}$, however, the imaginary part of this function (all we need of it) keeps finite at this point. So, the relation between $G_{y y}$ and $Z_{\text {rad }}$ can be written as: $G_{y y}\left(\mathbf{r}_{0}, \mathbf{r}_{0}, \omega\right)=4 j Z_{\mathrm{rad}} /\left(\omega \mu_{0} L\right)$. The impedance $Z_{\mathrm{rad}}$ of the short dipole is equal to its input impedance $Z_{\text {in }}$ if the dissipative losses are negligible compared to the radiative ones. ${ }^{1}$ Then, we have an expression which relates the imaginary part of Green's function of the environment of our dipole to its input resistance $R_{\text {in }} \equiv \operatorname{Re}\left[Z_{\text {in }}\right]: \operatorname{Im}\left[G_{y y}\left(\mathbf{r}_{0}, \mathbf{r}_{0}, \omega\right)\right]=4 R_{\text {in }} / \omega \mu_{0} L$. Writing this expression for a dipole located in the inhomogeneous environment and for the same a dipole in free space and taking the ratio of these expressions, we find

$$
\frac{R_{\mathrm{in}}}{R_{\mathrm{in}}^{0}}=\frac{\operatorname{Im}\left[G_{y y}\left(\mathbf{r}_{0}, \mathbf{r}_{0}, \omega\right)\right]}{\operatorname{Im}\left[G_{y y}^{0}\left(\mathbf{r}_{0}, \mathbf{r}_{0}, \omega\right)\right]}=\frac{P_{\mathrm{rad}}}{P_{\mathrm{rad}}^{0}},
$$

where $P_{\text {rad }}^{0}, R_{\text {in }}^{0}$, and $G_{y y}^{0}$ are, respectively, the radiated power and input impedance of the same dipole in free space, and Green's function of free space referred to the same point. So, the simulated Green's function can be used to find an optimal location of the dipole in an inhomogeneous environment that corresponds to maximal radiation. In our case, the inductive impedance induced by the notched resonator is not as sensitive to small offsets of the dipole as the induced resistance which plays the key role in the matching since $R_{\text {in }}^{0}$ is very small. The best matching practically corresponds to maximal $R_{\text {in }}$ which is achievable when the dipole is located inside the notch. In the pulse regime, the additional radiation resistance induced by the environment of the dipole means the shorter time of its oscillations following the pulse. This effect can be treated as the reduced lifetime of the excited state of the dipole. In photonics, this life-time reduction is called the Purcell effect. ${ }^{2,25,26}$ It is observed for many optical dipole sources, including quantum dots and atoms. In the so-called regime of weak coupling and in the absence of dissipation, their life-time reduction in presence of the environment is described by expression (3). Figure 5(a) shows the level of return losses (red curve) of our antenna obtained by numerical simulations. The value of return losses is smallest at $16.8 \mathrm{GHz}$ where the input resistance of the wire dipole equals to the wave impedance of the cable $(50 \Omega)$. Figure $5(\mathrm{~b})$ depicts return losses obtained experimentally. The agreement between the numerical results and experiment data is rather good. So, constructive mutual coupling of the dipole source with the dielectric resonator can be described in terms of better matching between the feeding cable and dipole source. This matching is not related to dissipative losses and we do not need additional matching/symmetrizing devices such as a "balun". 1 

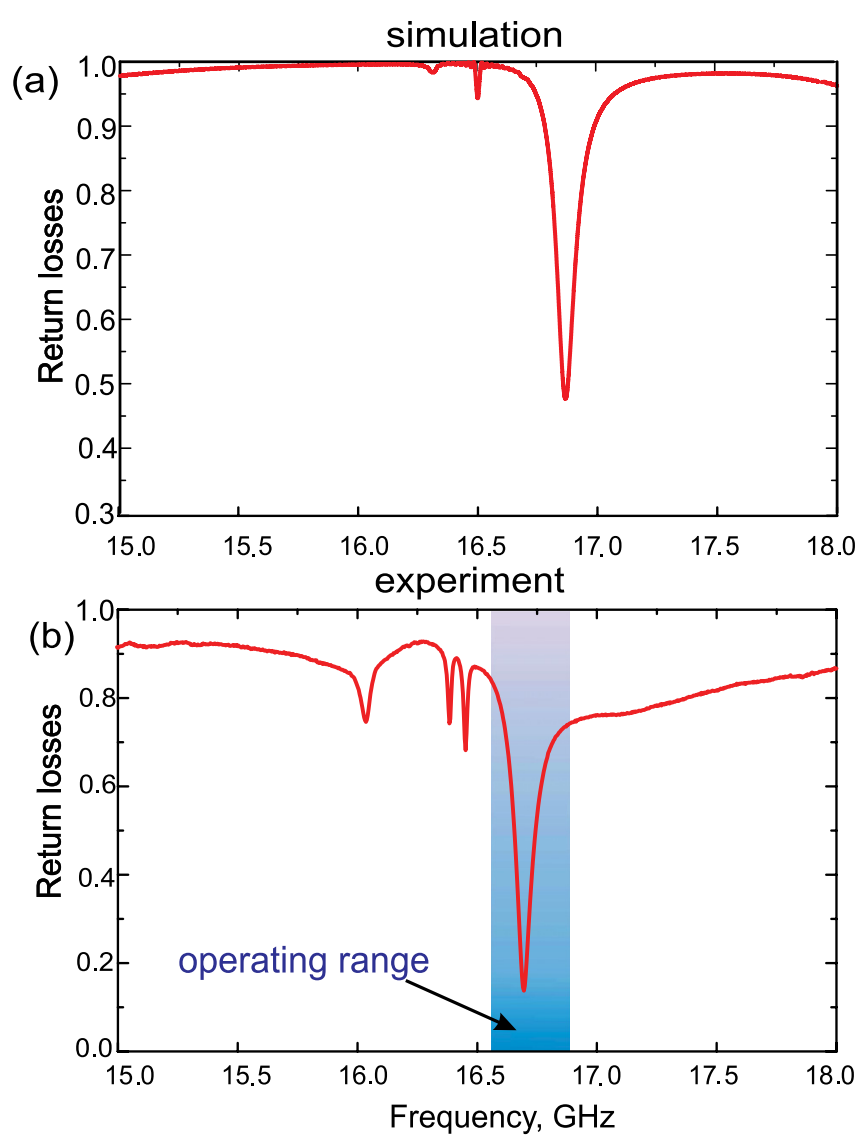

FIG. 5. (a) Level of return losses (red curve) and Purcell factor (blue curve) of the superdirective dielectric antenna obtained by numerical simulations. (b) Experimentally measured level of return losses of superdirective antenna. Blue area marks the operational frequency range where the directivity achieves its maximum.

In summary, we have suggested a simple approach for designing superdirective electrically small antennas, and confirmed this suggestion experimentally in the microwave frequency range. We have demonstrated the beam-steering effect through the subwavelength dipole displacement. We have shown that the good matching of a coaxial cable and a short wire dipole, without any special matching technique is due to a strong coupling of the dipole with the excited modes of the notched dielectric resonator. This matching is linked to the so-called Purcell effect. A combination of the superdirectivity, subwavelength beam steering, and low dissipation makes this type of antennas promising for numerous applications.
The authors thank E. A. Nenasheva and P. V. Kapitanova for a technical help. This work was supported by the Ministry of Education and Science of the Russian Federation (Project No. 11.G34.31.0020), the Russian Foundation for Basic Research, Dynasty Foundation (Russia), and the Australian National University.

${ }^{1}$ C. Balanis, Antenna Theory: Analysis and Design (Wiley, New York, 1982).

${ }^{2}$ A. E. Krasnok, I. S. Maksymov, A. I. Denisyuk, P. A. Belov, A. E. Miroshnichenko, C. R. Simovski, and Y. S. Kivshar, Phys.-Usp. 56, 539 (2013).

${ }^{3}$ L. Novotny and N. van Hulst, Nat. Photonics 5, 83 (2011).

${ }^{4}$ K. Fujimoto and H. Morishita, Modern Small Antennas (Cambridge University Press, 2013).

${ }^{5}$ E. H. Lim and K. W. Leung, Compact Multifunctional Antennas for Wireless Systems (John Wiley \& Sons, Inc., 2012).

${ }^{6}$ J. J. Adams, E. B. Duoss, T. F. Malkowski, M. J. Motala, B. Y. Ahn, R. G. Nuzzo, J. T. Bernhard, and J. A. Lewis, Adv. Mater. 23, 1335-1340 (2011).

${ }^{7}$ R. E. Hansen and R. C. Collin, Small Antenna Handbook (John Wiley and Sons Ltd., 2011).

${ }^{8}$ R. C. Hansen, Electrically Small, Superdirective, and Superconducting Antennas (Wiley-Interscience, 2006).

${ }^{9}$ A. D. Yaghjian, T. H. O’Donnell, E. E. Altshuler, and S. R. Best, Radio Sci. 43, RS3002, doi:10.1029/2007RS003747 (2008).

${ }^{10}$ N. Bikhazi and M. Jensen, IEEE Trans. Wireless Commun. 6, 1796 (2007).

${ }^{11}$ K. Itoh, O. lshii, Y. Nagai, N. Suzuki, Y. Kimachi, and O. Michikami, J. Supercond. 5, 485 (1992).

${ }^{12}$ R. J. Dinger, J. Supercond. 3, 287 (1990).

${ }^{13}$ I. I. Soloviev, V. K. Kornev, A. V. Sharafiev, N. V. Klenov, and O. A. Mukhanov, J. Surf. Invest. 6, 591 (2012).

${ }^{14}$ A. Alu and N. Engheta, IEEE Trans. Antennas Propag. 55, 3027 (2007).

${ }^{15}$ V. Mocella, P. Dardano, I. Rendina, and S. Cabrini, Opt. Express 18, 25068 (2010).

${ }^{16}$ A. Ludwig, C. D. Sarris, and G. V. Eleftheriades, Phys. Rev. Lett. 109, 223901 (2012).

${ }^{17}$ S. Enoch, G. Tayeb, P. Sabouroux, N. Guerin, and P. Vincent, Phys. Rev. Lett. 89, 213902 (2002).

${ }^{18}$ A. E. Krasnok, C. R. Simovski, P. A. Belov, and Y. S. Kivshar, "Superdirective dielectric nanoantenna," Nanoscale (submitted).

${ }^{19}$ M. V. Rybin, P. V. Kapitanova, D. S. Filonov, A. P. Slobozhanyuk, P. A. Belov, Y. S. Kivshar, and M. F. Limonov, Phys. Rev. B 88, 205106 (2013).

${ }^{20}$ B. Rolly, J.-M. Geffrin, R. Abdeddaim, B. Stout, and N. Bonod, Sci. Rep. 3, 3063 (2013).

${ }^{21}$ D. S. Filonov, A. E. Krasnok, A. P. Slobozhanyuk, P. V. Kapitanova, E. A. Nenasheva, Y. S. Kivshar, and P. A. Belov, Appl. Phys. Lett. 100, 201113 (2012).

${ }^{22}$ H. F. Ma and T. J. Cui, Nat. Commun. 1, 124 (2010).

${ }^{23}$ S. A. Tretyakov, S. Maslovski, and P. A. Belov, IEEE Trans. Antennas Propag. 51, 2652 (2003).

${ }^{24}$ J. Jackson, Classical Electrodynamics (Wiley, New York, 1998).

${ }^{25}$ D. Pohl, A. Alu, N. Engheta, and F. Marquier, Optical Antennas, edited by M. Agio and A. Alu (Cambridge University Press, 2013).

${ }^{26}$ L. Novotny and B. Hecht, Principles of Nano-Optics (Cambridge University Press, 2006). 